\title{
Electromagnetically induced transparency like transmission in a metamaterial composed of cut-wire pairs with indirect coupling
}

\author{
Yasuhiro Tamayama,,, Kanji Yasui, ${ }^{1}$ Toshihiro Nakanishi, ${ }^{2}$ and Masao Kitano ${ }^{2}$ \\ ${ }^{1}$ Department of Electrical Engineering, Nagaoka University of Technology, \\ 1603-1 Kamitomioka, Nagaoka, Niigata 940-2188, Japan \\ ${ }^{2}$ Department of Electronic Science and Engineering, Kyoto University, Kyoto 615-8510, Japan
}

(Dated: March 2, 2022)

\begin{abstract}
We theoretically and numerically investigate metamaterials composed of coupled resonators with indirect coupling. First, we theoretically analyze a mechanical model of coupled resonators with indirect coupling. The theoretical analysis shows that an electromagnetically induced transparency (EIT)-like phenomenon with a transparency bandwidth narrower than the resonance linewidths of the constitutive resonators can occur in the metamaterial with strong indirect coupling. We then numerically examine the characteristics of the metamaterial composed of coupled cut-wire pairs using a finite-difference time-domain (FDTD) method. The FDTD simulation confirms that an EIT-like transparency phenomenon occurs in the metamaterial owing to indirect coupling. Finally, we compare the results of the theoretical and numerical analyses. The behavior of the EIT-like metamaterial is found to be well described by the mechanical model of the coupled resonators.
\end{abstract}

PACS numbers: 78.67.Pt, 41.20.Jb, 78.20.Ci

\section{INTRODUCTION}

Metamaterials are arrays of artificial structures that are much smaller than the wavelength of electromagnetic waves. The macroscopic characteristics of metamaterials are determined by their constitutive elements and, therefore, electromagnetic media with desired properties can be created by designing the shape, material, and density of the constitutive elements. We can fabricate various media that do not occur in nature and can use such designed and fabricated metamaterials to control electromagnetic waves at will.

Resonant structures such as split-ring resonators $1 \frac{1}{\underline{1}}$ and electric-field-coupled inductor-capacitor resonators ${ }^{2}$ are typically used as constitutive elements of metamaterials. These structures are often called meta-atoms. The effective relative permittivity and permeability of metamaterials can be varied from unity and can even be made negative by using the strong response of these meta-atoms around the resonant frequencies. These resonant meta-atoms enable the realization of exotic phenomena such as negative refraction,,$\frac{3.4}{5}$ simultaneous negative phase and group velocities,,$\frac{5}{5}$ subwavelength imaging,,$\underline{6}$ and cloaking $\stackrel{7}{=}$ In addition, metamaterials composed of coupled resonators, which are often called metamolecules, have been investigated to realize useful characteristics such as giant optical activity ${ }^{-}$and enhancement of second-harmonic generation ${ }^{-9} \underline{\underline{11}}$ Dispersion control by introducing coupling between neighboring unit cells has also been studied. ${ }^{12,13}$

Although only electric and magnetic direct couplings (near-field couplings) have been introduced in the above mentioned metamaterials, indirect coupling mediated by radiative modes has been introduced in other coupledresonator systems. For example, indirect coupling has been introduced to achieve electromagnetically induced transparency (EIT)-like transmission in a two- dimensional photonic crystal waveguide coupled with two

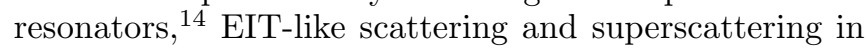
a double-slit structure in a metal film, $\frac{15}{5}$ and control of light at the nanoscale using plasmonic antennas $\frac{16}{\underline{16}}$ indirect coupling could be introduced into metamaterials as well as these isolated coupled-resonator systems, further development of methods for controlling electromagnetic waves could be expected. However, it is not obvious whether indirect coupling can be induced only between meta-atoms in each unit cell of metamaterials composed of periodically arranged coupled resonators (metamolecules).

In this paper, we analyze the characteristics of metamaterials composed of coupled resonators with indirect coupling. First, we use a mechanical model of coupled resonators to show that an EIT-like transparency phenomenon occurs when indirect coupling is introduced. The characteristics of the EIT-like metamaterial with indirect coupling are compared to those of previously investigated EIT-like metamaterials with direct coupling. $\underline{\underline{17}} \underline{\underline{23}}$ Then, we show through a finite-difference time-domain (FDTD) simulation 24 that the EIT-like transparency phenomenon caused by the indirect coupling is observed in the metamaterial composed of coupled cut-wire pairs. Finally, we discuss whether indirect coupling can be induced only between meta-atoms in each unit cell of metamaterials by comparing the theory based on the mechanical model and the results of the numerical analysis.

\section{MODELS OF COUPLED RESONATORS}

We analyze the electromagnetic response of a metamaterial composed of metamolecules that are modeled by the mechanical model shown in Fig.1(a). When the masses of the two particles are the same $\left(m_{1}=m_{2}=m\right)$ and the elastic constant of the central spring is much 
(a)

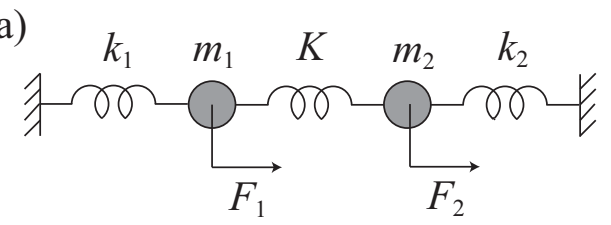

(b)

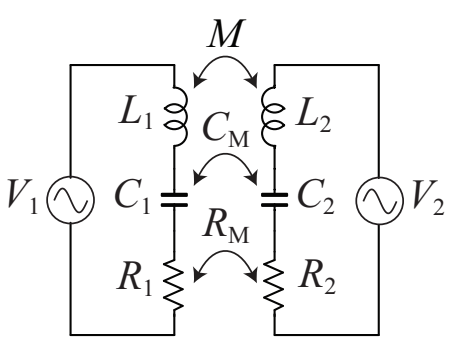

FIG. 1. (a) Mechanical model and (b) electrical circuit model of coupled resonators.

smaller than that of the other springs $\left(K \ll k_{1,2}\right)$, the equation of motion is given by

$$
\begin{aligned}
& \left(-\omega^{2}+\omega_{1}^{2}-\mathrm{i} \gamma_{1} \omega\right) x_{1}-\kappa^{2} x_{2}=\alpha_{1} E_{0}, \\
& \left(-\omega^{2}+\omega_{2}^{2}-\mathrm{i} \gamma_{2} \omega\right) x_{2}-\kappa^{2} x_{1}=\alpha_{2} E_{0},
\end{aligned}
$$

where $\omega_{1,2}=\sqrt{k_{1,2} / m}, \kappa=\sqrt{K / m}, \gamma_{1,2}$ are damping constants, and $F_{1,2}=m \alpha_{1,2} E_{0}$ are external forces, which are assumed to be proportional to the electric field $E_{0}$ of the electromagnetic wave with proportionality constants of $\alpha_{1,2}$. Solving Eqs. (11) and (21) for $x_{1}$ and $x_{2}$, the following equation is obtained:

$$
\begin{aligned}
{\left[\begin{array}{l}
x_{1} \\
x_{2}
\end{array}\right]=} & \frac{-1}{\left(\omega^{2}-\omega_{1}^{2}+\mathrm{i} \gamma_{1} \omega\right)\left(\omega^{2}-\omega_{2}^{2}+\mathrm{i} \gamma_{2} \omega\right)-\kappa^{4}} \\
& \times\left[\begin{array}{l}
\left(\omega^{2}-\omega_{2}^{2}+\mathrm{i} \gamma_{2} \omega\right) \alpha_{1}-\kappa^{2} \alpha_{2} \\
\left(\omega^{2}-\omega_{1}^{2}+\mathrm{i} \gamma_{1} \omega\right) \alpha_{2}-\kappa^{2} \alpha_{1}
\end{array}\right] E_{0} .
\end{aligned}
$$

We assume that the electric susceptibility of the metamaterial is proportional to $x_{1}+x_{2}$. We further assume that the characteristics of the two resonators are similar to each other and that particles 1 and 2 are coupled only via indirect coupling, that is, $\kappa^{2}$ is a purely imaginary quantity, $\kappa^{2}=\mathrm{i} \operatorname{Im}\left(\kappa^{2}\right)$. When $\gamma_{1,2}$ are derived only from the radiations of the meta-atoms, both $\gamma_{1,2}$ and $\alpha_{1,2}$ represent the coupling between the meta-atoms and freespace. Thus, $\alpha_{1} / \alpha_{2}=\gamma_{1} / \gamma_{2}$ can be safely assumed $\underline{16}$ Using the above assumptions, we obtain $x_{1}+x_{2}$ at around $\omega \simeq \omega_{0}$ as follows:

$$
\begin{aligned}
x_{1}+x_{2} \approx & -2 \alpha E_{0}\left(\omega^{2}-\omega_{0}^{2}+\mathrm{i} \gamma_{L} \omega\right) \\
& \times\left\{\left(\omega^{2}-\omega_{0}^{2}+\mathrm{i} \gamma_{1} \omega\right)\left(\omega^{2}-\omega_{0}^{2}+\mathrm{i} \gamma_{2} \omega\right)\right. \\
& \left.-\left(\omega_{0}^{2}-\omega_{1}^{2}\right)\left(\omega_{2}^{2}-\omega_{0}^{2}\right)+\left[\operatorname{Im}\left(\kappa^{2}\right)\right]^{2}\right\}^{-1},
\end{aligned}
$$

where $\omega_{0}^{2}=\left(\alpha_{2} \omega_{1}^{2}+\alpha_{1} \omega_{2}^{2}\right) /\left(\alpha_{1}+\alpha_{2}\right), \mathrm{i} 2 \gamma_{\mathrm{L}} \omega \alpha=\mathrm{i} \gamma_{2} \omega \alpha_{1}+$ $\mathrm{i} \gamma_{1} \omega \alpha_{2}-\left(\alpha_{1}+\alpha_{2}\right) \kappa^{2}$, and $\alpha=\left(\alpha_{1}+\alpha_{2}\right) / 2$. The righthand side of Eq. (4) resembles the susceptibility of the classical model of EIT with direct coupling $\underline{25}$ The correspondences of main parameters in the susceptibility of classical EIT with direct coupling to parameters in Eq. (41) are as follows: Both the resonant angular frequencies of the bright and dark modes are equal to $\omega_{0}$, the loss of the dark mode is nearly the same as $\gamma_{\mathrm{L}}$, and the coupling factor between the bright and dark modes corresponds to $\left(\omega_{0}^{2}-\omega_{1}^{2}\right)\left(\omega_{2}^{2}-\omega_{0}^{2}\right)-\left[\operatorname{Im}\left(\kappa^{2}\right)\right]^{2}$. This implies that an EIT-like transparency phenomenon occurs at the angular frequency $\omega=\omega_{0} \approx\left(\omega_{1}+\omega_{2}\right) / 2$. Note that $\gamma_{\mathrm{L}}$ becomes less than $\gamma_{1,2}$ if $\operatorname{Im}\left(\kappa^{2}\right)>0$. From the passive condition for the metamaterial, the imaginary part of $x_{1}+x_{2}$ must be non-negative. To satisfy the passive condition at $\omega=\omega_{0}$ irrespective of $\omega_{1}$ and $\omega_{2}, \operatorname{Im}\left(\kappa^{2}\right) \leq \omega_{0} \sqrt{\gamma_{1} \gamma_{2}}$ is required.

Next, we consider the group index at $\omega=\omega_{0}$. When the absolute value of $\Delta=\omega_{1}-\omega_{2}$ is larger than a certain value $\Delta_{\max }$, the transmission bandwidth decreases; that is, the group index increases with decreasing $|\Delta|$. When $|\Delta|$ is smaller than $\Delta_{\max }$, the transmission window gradually disappears and the group index decreases with decreasing $|\Delta|$ due to the loss. The value of $\Delta_{\max }$ can be regarded as the minimum transmission bandwidth. We calculate $\Delta_{\max }$ that gives the condition for the largest group index below.

We may assume that the value of $|\Delta|$ that maximizes $\operatorname{Re}\left[\mathrm{d}\left(x_{1}+x_{2}\right) /\left.\mathrm{d} \omega\right|_{\omega=\omega_{0}}\right]$ is almost equal to $\Delta_{\max }$ in the case of strongly dispersive media as in the present case. For simplicity of the analysis, we further assume that $\gamma_{1}=\gamma_{2}=\gamma_{0}$, from which $\operatorname{Im}\left(\kappa^{2}\right)=\omega_{0}\left(\gamma_{0}-\gamma_{\mathrm{L}}\right)$ is obtained. This implies that $\gamma_{\mathrm{L}}$ that appears in Eq. (4) represents the leak of the indirect coupling. From Eq. (44), we obtain

$$
\operatorname{Re}\left[\left.\frac{\mathrm{d}\left(x_{1}+x_{2}\right)}{\mathrm{d} \omega}\right|_{\omega=\omega_{0}}\right] \approx \frac{4 \alpha E_{0}\left(\Delta^{2}-\gamma_{\mathrm{L}}^{2}\right)}{\omega_{0}\left(\Delta^{2}+2 \gamma_{0} \gamma_{\mathrm{L}}-\gamma_{\mathrm{L}}^{2}\right)^{2}} .
$$

The right-hand side takes a maximum value for $|\Delta|=$ $\sqrt{2 \gamma_{0} \gamma_{\mathrm{L}}+\gamma_{\mathrm{L}}^{2}}$, which we define as $\Delta_{\max }$. In the case of $\gamma_{\mathrm{L}}=0$, the transmission bandwidth can become infinitesimal and the group index can become infinite. Note that the transmission bandwidth can be smaller than the resonance linewidth $\gamma_{0}$ of the meta-atoms when the leak $\gamma_{\mathrm{L}}$ of the indirect coupling is sufficiently small. It is also found from Eq. (5) that the group index becomes negative; that is, the transmission window disappears in the range $|\Delta|<\gamma_{\mathrm{L}}$.

Here we discuss the derivation of the leak $\gamma_{\mathrm{L}}$ of the indirect coupling, which is an important parameter that determines the minimum bandwidth of the transparency window. Since the indirect coupling is mediated by radiative modes, the indirect coupling takes a maximum value, that is, $\gamma_{\mathrm{L}}$ vanishes when all the energy dissipated from one meta-atom in each unit cell is absorbed by the other meta-atom. The dissipated energy is derived from Ohmic loss, dielectric loss, and radiative loss in most meta-atoms. The dissipated energy derived from Ohmic and dielectric losses cannot excite the other meta-atom, 
and thus, Ohmic and dielectric losses contribute to $\gamma_{\mathrm{L}}$. In addition, the difference between the radiation modes of the two kinds of meta-atoms causes a reduction of the radiative coupling, i.e., an increase in $\gamma_{\mathrm{L}}$. Thus, $\gamma_{\mathrm{L}}$ is derived from Ohmic loss, dielectric loss, and the difference between the radiation modes of the two kinds of meta-atoms.

We also discuss the physical meaning of the narrower transmission bandwidth than the resonance linewidths of the meta-atoms in the transparency phenomenon. For $\omega=\omega_{0}$, Eq. (4) is reduced to $x_{1} \approx-x_{2}$. In this case, the dissipation terms derived from $\gamma_{1,2}$ in Eqs. (11) and (2) are (partially) canceled out by the terms derived from the indirect coupling $\kappa^{2}=\mathrm{i} \operatorname{Im}\left(\kappa^{2}\right)$. This implies that the energy radiated from one meta-atom in each unit cell is absorbed by the other meta-atom. That is, the radiated energy moves backward and forward between the two kinds of meta-atoms in each unit cell. Therefore, the effective radiation loss in the metamolecule is reduced and the narrowband transparency phenomenon can be achieved.

We assumed $\operatorname{Re}\left(\kappa^{2}\right)=0$ in the above discussion. In the case of $\operatorname{Re}\left(\kappa^{2}\right) \neq 0$, it is found from Eq. (3) that the EIT-like transparency phenomenon occurs for $\omega^{2}=$ $\omega_{0}^{2}+\operatorname{Re}\left(\kappa^{2}\right)$.

It is useful for understanding the electromagnetic response of the metamaterial to analyze an electrical circuit model of the metamolecule. We consider an electrical circuit that consists of two coupled inductor-capacitor resonant circuits, shown in Fig.1(b). Three kinds of couplings exist in the electrical circuit. Applying Kirchhoff's voltage law for the electrical circuit yields the following equations:

$$
\begin{aligned}
& \left(-\omega^{2}+\frac{1}{L C_{1}}-\mathrm{i} \frac{R_{1}}{L} \omega\right) q_{1} \\
& -\frac{\mathrm{i} \omega}{L}\left[R_{\mathrm{M}}-\mathrm{i}\left(\omega M-\frac{1}{\omega C_{\mathrm{M}}}\right)\right] q_{2}=\frac{V_{1}}{L}, \\
& \left(-\omega^{2}+\frac{1}{L C_{2}}-\mathrm{i} \frac{R_{2}}{L} \omega\right) q_{2} \\
& -\frac{\mathrm{i} \omega}{L}\left[R_{\mathrm{M}}-\mathrm{i}\left(\omega M-\frac{1}{\omega C_{\mathrm{M}}}\right)\right] q_{1}=\frac{V_{2}}{L},
\end{aligned}
$$

where $L_{1}=L_{2}=L$ is assumed. It is found by comparing Eqs. (11) and (2) with Eqs. (6) and (77) that the imaginary and real parts of $\kappa^{2}$ correspond to the real and imaginary parts, respectively, of the mutual impedance $Z_{\mathrm{M}}=R_{\mathrm{M}}-$ $\mathrm{i}\left[\omega M-\left(\omega C_{\mathrm{M}}\right)^{-1}\right]$. This relation shows the influences of the electric coupling $C_{\mathrm{M}}$, magnetic coupling $M$, and energy coupling $R_{\mathrm{M}}$ on $\kappa^{2}$.

We now discuss the difference between the EIT-like metamaterial with indirect coupling and that with direct coupling. $17-23$ The unit cell of the latter metamaterial consists of two directly coupled meta-atoms: a low quality-factor meta-atom, which interacts with the incident electromagnetic wave, and a high quality-factor meta-atom, which does not interact with the incident wave. The loss in the high quality-factor meta-atom

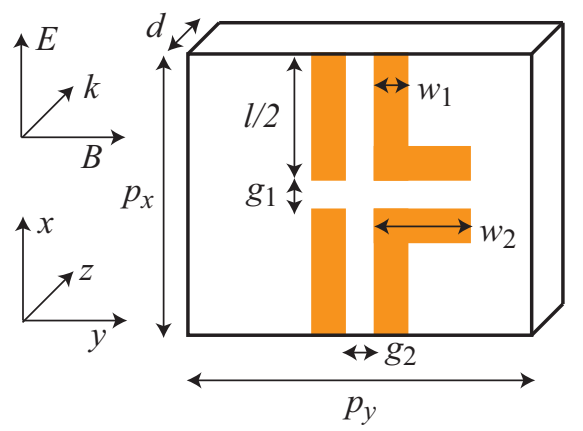

FIG. 2. Unit cell of the EIT-like metamaterial with indirect coupling. A thin metallic film represented by brown (gray) lies on a dielectric substrate represented by white.

should be reduced to achieve a large group index. The resonant frequencies of the two kinds of meta-atoms should be identical to ensure that the transmission spectrum is symmetric to reduce higher-order dispersion. On the other hand, as described above, the unit cell of the former metamaterial consists of two similar meta-atoms. The indirect coupling should be strong to realize a large group index. The two meta-atoms should be coupled so that $\operatorname{Re}\left(\kappa^{2}\right)=0$ is satisfied to ensure that the transmission spectrum is symmetric. Both kinds of EIT-like metamaterials require efforts to achieve a large group index and symmetric transmission spectrum. However, the structure of the meta-atoms can be simple for the former metamaterial, because the unit cell consists of two similar meta-atoms and the radiation losses of the meta-atoms may be large. Therefore, the former metamaterial can be superior in terms of ease of design and fabrication to the latter metamaterial if $\gamma_{\mathrm{L}} \simeq 0$ and $\operatorname{Re}\left(\kappa^{2}\right)=0$ are simultaneously satisfied.

\section{FDTD ANALYSIS OF EIT-LIKE METAMATERIAL WITH INDIRECT COUPLING}

In order to confirm the validity of the above theory and to investigate whether $\gamma_{\mathrm{L}} \simeq 0$ and $\operatorname{Re}\left(\kappa^{2}\right)=0$ are simultaneously satisfied, we design the EIT-like metamaterial with indirect coupling and analyze the characteristics of the metamaterial using an FDTD method $\stackrel{24}{2}$ Figure 2 shows the unit cell of the EIT-like metamaterial whose electromagnetic response is modeled by the mechanical model shown in Fig.1(a). Two kinds of cut-wire resonators (meta-atoms) with different resonant frequencies are placed with a gap of $g_{2}$. The structures of the two kinds of cut-wire resonators are designed to be similar to each other in order to make their characteristics including the radiation modes similar. The configuration of the two kinds of cut-wire resonators is determined so that the radiation from one cut-wire resonator can excite the other cut-wire resonator; that is, indirect coupling is induced in the cut-wire pair. The unit structure can be re- 


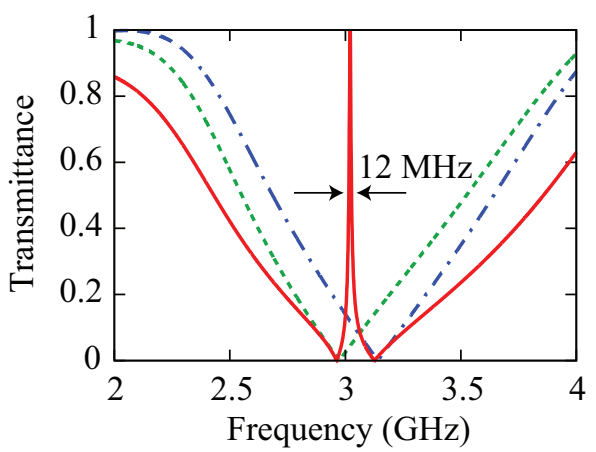

FIG. 3. Transmission spectra for the lossless substrate. The red solid curve represents the transmission spectrum of the metamaterial composed of coupled cut-wire pairs. The green dashed curve and blue dashed-dotted curve represent the transmission spectra of the metamaterials composed of one kind of cut wires.

garded as a kind of asymmetric split-ring resonator $\stackrel{26,27}{\underline{1}}$ The resonant frequencies, $\omega_{1}$ and $\omega_{2}$, of the two kinds of resonators are determined by the inductance derived from the metal wire with length $l$ and the capacitance derived from the gap $g_{1}$. The difference $|\Delta|$ of the resonant angular frequencies between the two kinds of resonators is determined by the difference between $w_{1}$ and $w_{2}$. The resonance linewidths, $\gamma_{1}$ and $\gamma_{2}$, of the two kinds of resonators depend on the radiation loss, dielectric loss in the substrate, and Ohmic loss in the metal. The coupling factor $\kappa$ between the two kinds of resonators can be controlled by varying $g_{2}$. The FDTD simulation is performed in the microwave region where metals can be regarded as perfect electric conductors; that is, Ohmic loss in the metal is negligible. This enables us to investigate the influence of the leak $\gamma_{\mathrm{L}}$ of the indirect coupling on the transmission characteristics by only varying the dielectric loss of the substrate and to easily compare the theory based on the mechanical model with the results of the FDTD analysis.

We first calculated the transmission spectrum of the EIT-like metamaterial. The geometrical parameters of the metamaterial were set to $l=28 \mathrm{~mm}, w_{1}=3 \mathrm{~mm}$, $w_{2}=4 \mathrm{~mm}, g_{1}=2 \mathrm{~mm}, g_{2}=2 \mathrm{~mm}, d=1 \mathrm{~mm}$, $p_{x}=29 \mathrm{~mm}$, and $p_{y}=39 \mathrm{~mm}$. The relative permittivity of the substrate was set to 3.3 , which is the real part of the relative permittivity of polyphenylene ether. The FDTD simulation space was discretized into uniform cubes with dimensions of $1 \mathrm{~mm} \times 1 \mathrm{~mm} \times 1 \mathrm{~mm}$. (Although the thickness of the substrate was modeled using only a single FDTD cell in the simulation, no significant errors were caused to the simulation results.) Periodic boundary conditions were applied to the $x$ and $y$ directions to realize periodically arranged metamolecules.

Figure 3 shows the transmission spectra of three kinds of metamaterials. The red solid curve represents the transmission spectrum of the metamaterial composed of coupled cut-wire pairs (metamolecules) described above. (a)

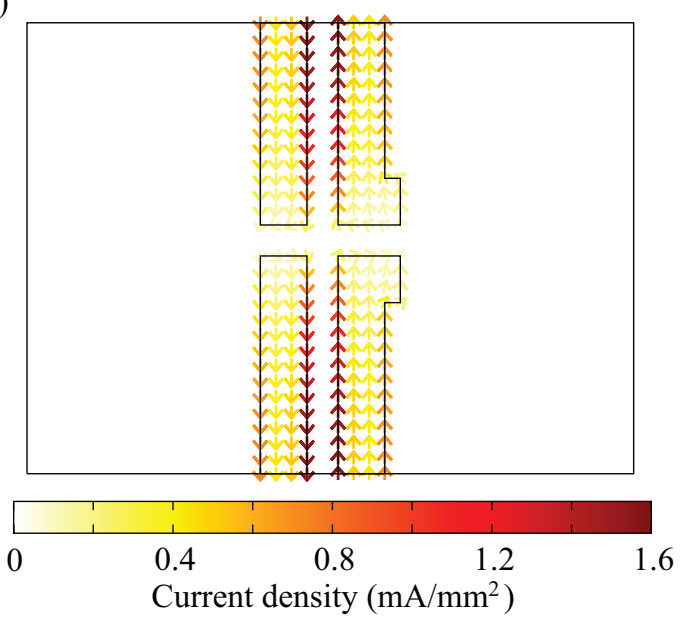

(b)

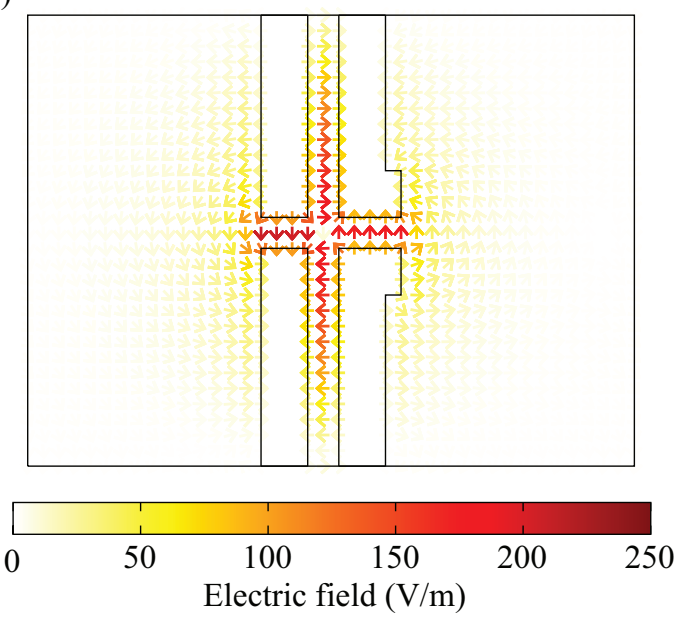

FIG. 4. Snapshots of (a) the current and (b) electric field distributions at the transparency frequency. The incident electric field is $1 \mathrm{~V} / \mathrm{m}$.

The green dashed curve (blue dashed-dotted curve) represents the transmission spectrum of the metamaterial composed of one kind of cut wires, i.e., meta-atoms, shown in the right-hand side (left-hand side) of Fig.2. While simple absorption spectra are observed for the metamaterials composed of one kind of cut wires, a transmission window is observed in the absorption spectrum for the metamaterial composed of coupled cut-wire pairs. In addition, the transmission bandwidth is much smaller than the resonance linewidths (absorption bandwidths) of the metamaterials composed of one kind of cut wires. This implies that the EIT-like transparency phenomenon occurs for the metamaterial composed of coupled cutwire pairs owing to the indirect coupling. Since the transmittance at the transparency frequency is nearly unity, $\gamma_{\mathrm{L}}$ seems to be negligibly small for the present geometrical parameters. Although the present metamaterial, which is composed of periodically arranged coupled resonators, is different from isolated coupled resonators such 


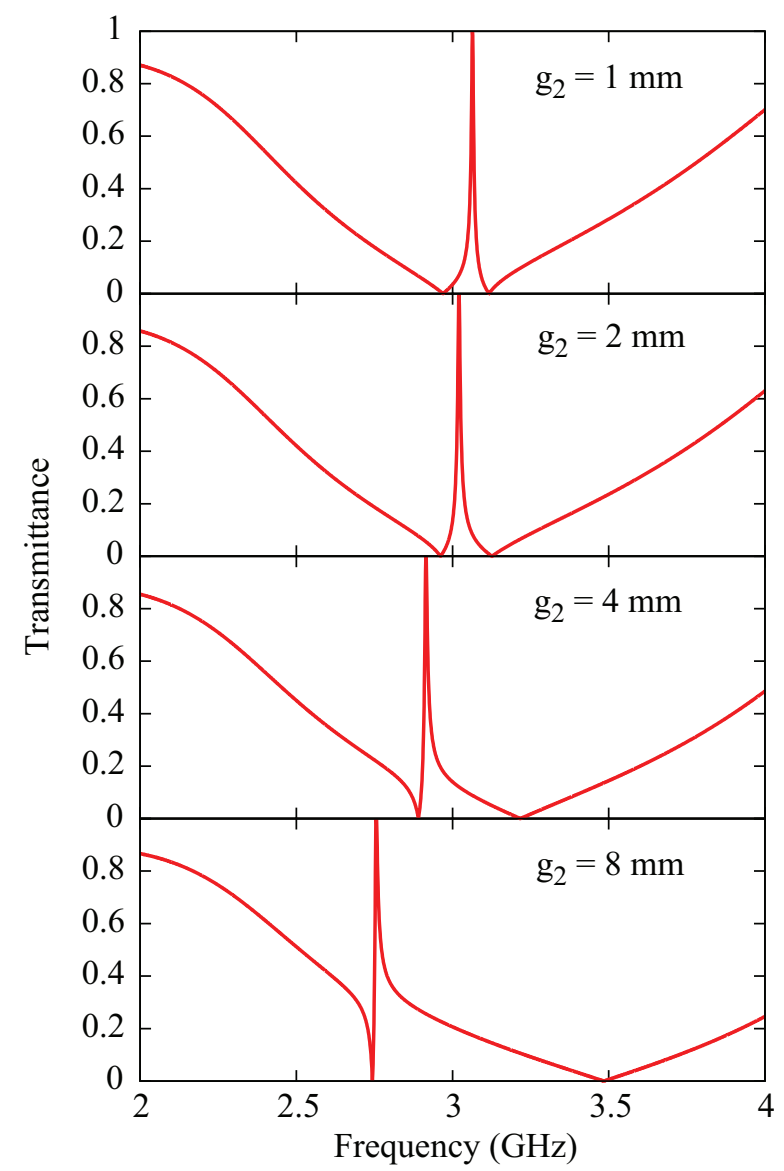

FIG. 5. Dependence of the transmission spectrum on $g_{2}$.

as the mechanical model shown in Fig.11(a) and the coupled resonators in previous studies,, $14-16$ indirect coupling can be induced between the meta-atoms in each unit cell and $\gamma_{\mathrm{L}}$ seems to be negligibly small also in the metamaterial. (Note that it is still unclear whether indirect coupling is induced between different cells.)

We next calculated the field distributions at the transparency frequency to understand the physical meaning of the EIT-like transparency phenomenon. Figure 4(a) shows the current distribution at the transparency frequency. An antisymmetric current flows in the coupled cut-wire pair and thus the total electric dipole moment vanishes. That is, the scattering is suppressed due to destructive interference between the radiations from the two kinds of cut wires. This observation is another aspect of the cancellation between the radiation loss and the indirect coupling described in Sec. II. Figure 4(b) shows the electric field distribution at the transparency frequency. A large quadrupole electric field is induced at the gap of the two kinds of cut wire. The electric field at the gap is about 200 times as large as the incident electric field, in which the narrow band effect is reflected.

We next analyzed the dependence of the transmission spectrum on $g_{2}$ to investigate whether $\operatorname{Re}\left(\kappa^{2}\right)=0$ can

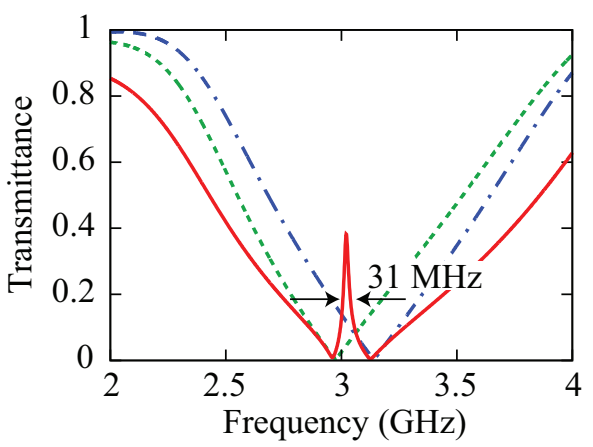

FIG. 6. Transmission spectra for $\tan \delta=0.005$. The red solid curve represents the transmission spectrum of the metamaterial composed of coupled cut-wire pairs. The green dashed curve and blue dashed-dotted curve represent the transmission spectra of the metamaterials composed of one kind of cut wires.

be satisfied by varying $g_{2}$. Figure 5 shows the transmission spectra for $g_{2}=1 \mathrm{~mm}, 2 \mathrm{~mm}, 4 \mathrm{~mm}$, and $8 \mathrm{~mm}$. The other parameters are the same as those in the case of Fig.3. With increasing $g_{2}$, the transmission peak shifts to lower frequency; that is, $\operatorname{Re}\left(\kappa^{2}\right)$ decreases. The transmission spectrum for $g_{2}=1 \mathrm{~mm}$ shows the opposite asymmetry to that for $g_{2}=2 \mathrm{~mm}$ and, therefore, the condition that satisfies $\operatorname{Re}\left(\kappa^{2}\right)=0$ exists in the range $1 \mathrm{~mm}<g_{2}<2 \mathrm{~mm}$. The imaginary part of $\kappa^{2}$ seems to be a constant value in the range $1 \mathrm{~mm}<g_{2}<8 \mathrm{~mm}$ because the transmittance at the transparency frequency is nearly unity for these four calculated conditions.

The dependence of $\operatorname{Re}\left(\kappa^{2}\right)$ on $g_{2}$ can be understood using the electrical circuit model of the coupled resonators shown in Fig.1(b). As $g_{2}$ increases, both the mutual inductance $M$ and mutual capacitance $C_{\mathrm{M}}$ decrease and thus the imaginary part of the mutual impedance $Z_{\mathrm{M}}$ decreases. Therefore, $\operatorname{Re}\left(\kappa^{2}\right)$ decreases and the transmission peak shifts to lower frequency with increasing $g_{2}$. Note that this discussion can be applied only to the case of $g_{2} \ll \lambda$, where $\lambda$ is the wavelength of the electromagnetic waves. When $g_{2}$ is comparable to or larger than $\lambda$, the phase retardation of the coupling has to be taken into account; therefore, we cannot discuss the indirect and direct couplings separately. However, $g_{2} \ll \lambda$ is safely satisfied in metamaterials.

We next analyzed the influence of the dielectric loss of the substrate on the transmission characteristics. Figure 6] shows the transmission spectra of the above mentioned three kinds of metamaterials when the loss tangent $\tan \delta$ of the dielectric substrate is 0.005 , which is the loss tangent of polyphenylene ether at $3 \mathrm{GHz}$. The other parameters are the same as those in the case of Fig. 3. While the transmission spectra of the metamaterials composed of one kind of cut wires are almost the same as those in Fig.3, the transmittance of the metamaterial composed of coupled cut-wire pairs at the transparency frequency is smaller than that in the case of Fig. 3 due to the di- 
(a)

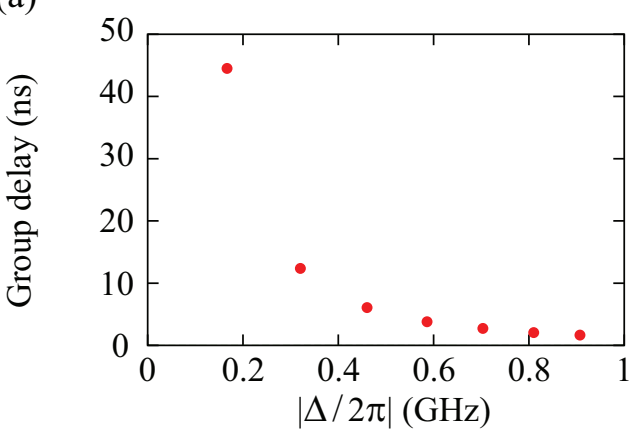

(b)

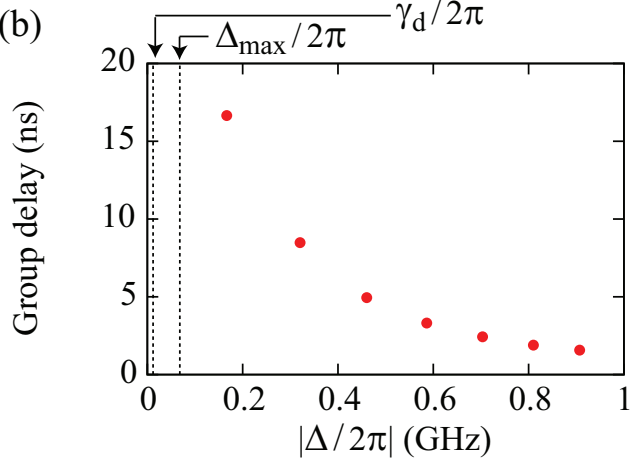

(c)
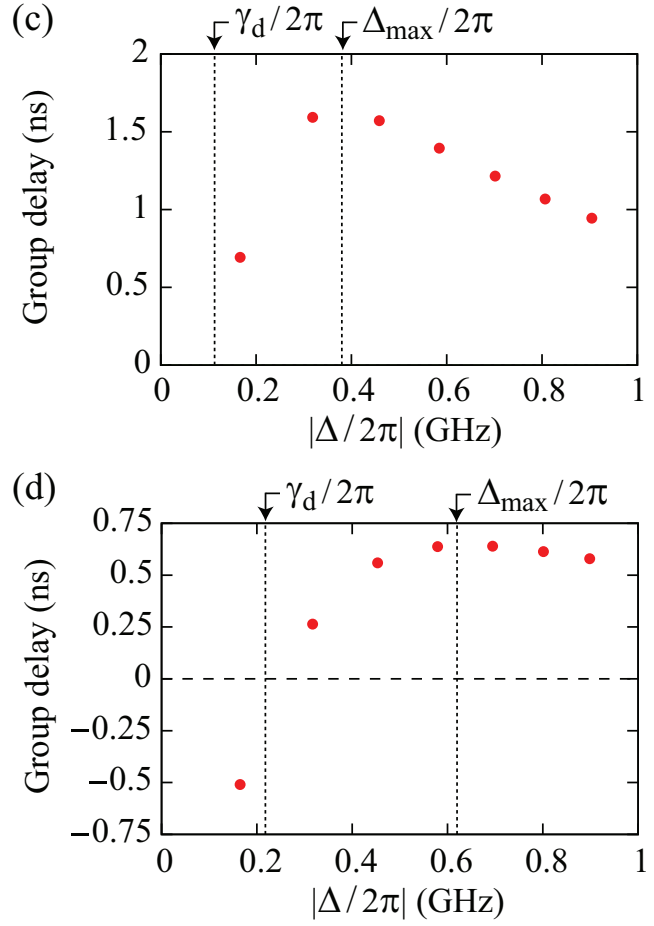

FIG. 7. Dependence of the group delay at the transparency frequency on $|\Delta|$ when $\tan \delta$ is equal to (a) 0 , (b) 0.005 , (c) 0.05 , and (d) 0.1 . The values of $\Delta_{\max }$ and $\gamma_{\mathrm{d}}$ are represented by the vertical dashed lines in each case. Note that the scales of the vertical axes are different from each other. electric loss. This implies that the leak of the indirect coupling without dielectric loss is much smaller than the dielectric loss $\gamma_{\mathrm{d}}$ of the substrate. Thus, we may assume $\gamma_{\mathrm{L}} \simeq \gamma_{\mathrm{d}}$ when $\tan \delta$ is larger than 0.005 at most.

Finally, we calculated the dependence of the group delay at the transparency frequency on $|\Delta|$ to investigate the influence of $\tan \delta$ on $\Delta_{\max }$. The absolute value of $\Delta$ was varied by varying $w_{2}$ from $4 \mathrm{~mm}$ to $10 \mathrm{~mm}$ with steps of $1 \mathrm{~mm}$. The other parameters except $\tan \delta$ are the same as those in the case of Fig.3.

Figure 7 shows the group delay at the transparency frequency as a function of $|\Delta|$ for $\tan \delta=0,0.005,0.05$, and 0.1 . For the calculated conditions, the group delay monotonically increases with decreasing $|\Delta|$ for $\tan \delta=0$ and 0.005 , while the group delay first increases and then decreases with decreasing $|\Delta|$ for $\tan \delta=0.05$ and 0.1 .

We now discuss the above results using the mechanical model of the coupled resonator shown in Fig.1(a). We have to evaluate in advance the radiation loss $\gamma_{0}$ of the cut-wire resonators and the dielectric loss $\gamma_{\mathrm{d}}$ of the substrate in order to use the mechanical model. For a Lorentz medium with a simple absorption line, $\gamma_{0}$ is almost equal to the bandwidth of the negative group delay. Thus, $\gamma_{0}$ can be estimated by calculating the group delay of the metamaterial composed of one kind of cut wires (not shown). The value of $\gamma_{\mathrm{d}}$ can be estimated as follows. In a series inductor-capacitor resonant circuit, the quality factor of the circuit can be approximated as the inverse of the loss tangent of the dielectric in the capacitor when the loss in the circuit is caused only by the dielectric loss in the capacitor. Since the thin metallic film is on the dielectric substrate in the metamaterial shown in Fig.2. we assume that half of the capacitor in the metamaterial is filled with the dielectric and the other half is filled with vacuum. That is, the capacitance of the gap is assumed to be $\left(1+\varepsilon_{\mathrm{r}}\right) / 2$ times as large as that without the substrate, where $\varepsilon_{\mathrm{r}}$ is the relative permittivity of the substrate. From this assumption, the effective loss tangent $\tan \delta^{\prime}$, which is the loss tangent when the capacitor is assumed to be filled with a uniform medium, is found to be $\left\{\operatorname{Re}\left(\varepsilon_{\mathrm{r}}\right) /\left[1+\operatorname{Re}\left(\varepsilon_{\mathrm{r}}\right)\right]\right\} \tan \delta$. Therefore, the dielectric loss $\gamma_{\mathrm{d}}$ in the metamaterial is estimated to be $\omega_{0} \tan \delta^{\prime}$.

We now show $\Delta_{\max }$ calculated using the above estimated $\gamma_{0}$ and $\gamma_{\mathrm{d}}$ in Fig. 7 The value of $\gamma_{\mathrm{d}}$ is also shown in the figure. It is found that the group delay increases or decreases with decreasing $|\Delta|$ when $|\Delta|$ is larger or smaller, respectively, than $\Delta_{\max }$ and that the group delay seems to vanish at around $|\Delta|=\gamma_{\mathrm{d}}$. This implies that the behavior of the coupled cut-wire pair metamaterial can be well described by the mechanical model. That is, indirect coupling can be induced only between meta-atoms in each unit cell of metamaterials composed of periodically arranged coupled resonators. 


\section{CONCLUSION}

We analyzed the EIT-like transparency phenomenon in metamaterials composed of coupled resonators with indirect coupling. The theoretical analysis based on the mechanical model showed that the transparency bandwidth can be narrower than the resonance linewidths of the constitutive resonators when strong indirect coupling is introduced. The FDTD simulation demonstrated that the EIT-like transparency phenomenon with $\gamma_{\mathrm{L}} \simeq 0$ and $\operatorname{Re}\left(\kappa^{2}\right)=0$ can occur in metamaterials composed of coupled cut-wire pairs. The characteristics of the metamaterial was confirmed to be well described by the mechanical model, and indirect coupling was found to be induced only between meta-atoms in each unit cell of metamaterials composed of periodically arranged coupled resonators.

Structures of meta-atoms with narrow resonance linewidth usually have complicated shapes. However, the narrow band transparency window can be obtained using simple structures such as cut wires when indirect cou- pling is introduced. If dielectric loss is prevented completely, an extremely narrow transparency window can be achieved below infrared frequencies where metals can be regarded as perfect electric conductors. For future studies, the minimum transparency bandwidth of the EITlike metamaterial without dielectric loss needs to be investigated experimentally. In the optical region, metals exhibit relatively large Ohmic losses, and thus the metamaterial should be designed with low-loss dielectrics. Indirect coupling would be useful not only for realizing EITlike phenomena but also for other techniques for controlling electromagnetic waves.

\section{ACKNOWLEDGMENTS}

This research was supported by a Grant-in-Aid for Scientific Research on Innovative Areas (No. 22109004) from the Ministry of Education, Culture, Sports, Science, and Technology, Japan, and by a Grant-in-Aid for Research Activity Start-up (No. 25889028) from the Japan Society for the Promotion of Science.
* tamayama@vos.nagaokaut.ac.jp

1 J. B. Pendry, A. J. Holden, D. J. Robbins, and W. J. Stewart, IEEE Trans. Microwave Theory Tech. 47, 2075 (1999).

2 D. Schurig, J. J. Mock, and D. R. Smith, Appl. Phys. Lett. 88, 041109 (2006).

3 R. A. Shelby, D. R. Smith, and S. Schultz, Science 292, 77 (2001).

4 J. Valentine, S. Zhang, T. Zentgraf, E. Ulin-Avila, D. A. Genov, G. Bartal, and X. Zhang, Nature 455, 376 (2008).

${ }^{5}$ G. Dolling, C. Enkrich, M. Wegener, C. M. Soukoulis, and S. Linden, Science 312, 892 (2006).

6 A. N. Lagarkov and V. N. Kissel, Phys. Rev. Lett. 92, 077401 (2004).

7 D. Schurig, J. J. Mock, B. J. Justice, S. A. Cummer, J. B. Pendry, A. F. Starr, and D. R. Smith, Science 314, 977 (2006).

${ }^{8}$ H. Liu, D. A. Genov, D. M. Wu, Y. M. Liu, Z. W. Liu, C. Sun, S. N. Zhu, and X. Zhang, Phys. Rev. B 76, 073101 (2007).

9 T. Kanazawa, Y. Tamayama, T. Nakanishi, and M. Kitano, Appl. Phys. Lett. 99, 024101 (2011).

10 T. Nakanishi, Y. Tamayama, and M. Kitano, Appl. Phys. Lett. 100, 044103 (2012).

11 R. Czaplicki, H. Husu, R. Siikanen, J. Mäkitalo, M. Kauranen, J. Laukkanen, J. Lehtolahti, and M. Kuittinen, Phys. Rev. Lett. 110, 093902 (2013).

12 E. Shamonina, Phys. Stat. Sol. (b) 245, 1471 (2008).

13 Y. Nakata, T. Okada, T. Nakanishi, and M. Kitano, Phys. Rev. B 85, 205128 (2012).

14 W. Suh, Z. Wang, and S. Fan, IEEE J. Quantum Electron. 40, 1511 (2004).
${ }^{15}$ L. Verslegers, Z. Yu, Z. Ruan, P. B. Catrysse, and S. Fan, Phys. Rev. Lett. 108, 083902 (2012).

16 S. Zhang, Z. Ye, Y. Wang, Y. Park, G. Bartal, M. Mrejen, X. Yin, and X. Zhang, Phys. Rev. Lett. 109, 193902 (2012).

17 S. Zhang, D. A. Genov, Y. Wang, M. Liu, and X. Zhang, Phys. Rev. Lett. 101, 047401 (2008).

18 P. Tassin, L. Zhang, T. Koschny, E. N. Economou, and C. M. Soukoulis, Phys. Rev. Lett. 102, 053901 (2009).

19 N. Liu, L. Langguth, T. Weiss, J. Kästel, M. Fleischhauer, T. Pfau, and H. Giessen, Nature Mater. 8, 758 (2009).

20 Y. Tamayama, T. Nakanishi, Y. Wakasa, T. Kanazawa, K. Sugiyama, and M. Kitano, Phys. Rev. B 82, 165130 (2010).

21 L. Zhang, P. Tassin, T. Koschny, C. Kurter, S. M. Anlage, and C. M. Soukoulis, Appl. Phys. Lett. 97, 241904 (2010).

${ }^{22}$ C. Kurter, P. Tassin, L. Zhang, T. Koschny, A. P. Zhuravel, A. V. Ustinov, S. M. Anlage, and C. M. Soukoulis, Phys. Rev. Lett. 107, 043901 (2011).

23 Y. Tamayama, T. Nakanishi, and M. Kitano, Phys. Rev. B 85, 073102 (2012).

24 A. Taflove and S. C. Hagness, Computational Electrodynamics: The Finite-Difference Time-Domain Method, 3rd ed. (Artech House, Norweed, MA, 2005).

25 C. L. Garrido Alzar, M. A. G. Martinez, and P. Nussenzveig, Am. J. Phys. 70, 37 (2002).

26 V. A. Fedotov, M. Rose, S. L. Prosvirnin, N. Papasimakis, and N. I. Zheludev, Phys. Rev. Lett. 99, 147401 (2007).

27 E. Plum, X.-X. Liu, V. A. Fedotov, Y. Chen, D. P. Tsai, and N. I. Zheludev, Phys. Rev. Lett. 102, 113902 (2009). 